\title{
Isolation and Absolute Configuration of SMTP-0, a Simplest Congener of the SMTP Family Nonlysine-analog Plasminogen Modulators
}

\author{
Keiji Hasumi, Keiko Hasegawa, Yoshikazu Kitano
}

Received: April 16, 2007 / Accepted: July 12, 2007

(C) Japan Antibiotics Research Association

\begin{abstract}
SMTP-0, a new simple congener of the SMTP nonlysine-analog plasminogen modulators, was isolated from a culture of Stachybotrys microspora. Based on the physico-chemical data, SMTP-0 was shown to be an enantiomer of the antimicrobial compound stachybotrin B. The absolute configuration of SMTP-0 was determined by the modified Mosher method. The stereochemistry was further confirmed using an epimer of SMTP-0. Unlike most SMTPs with an amino acid side chain linked to the nitrogen atom of the lactam moiety, SMTP-0, which lacks the $N$-linked side chain, showed no plasminogen modulator activity.
\end{abstract}

Keywords nonlysine-analog plasminogen modulator, absolute configuration, Stachybotrys microspora, microbial triprenyl phenols, modified Mosher method

The plasminogen/plasmin system plays crucial roles not only in blood clot lysis but also in various physiological and pathological events, where localized proteolysis is required [1, 2]. Human plasminogen is a single-chain glycoprotein consisting of an $\mathrm{N}$-terminal peptide, five kringle domains and a serine protease domain [3]. Two serine proteases, tissue-type plasminogen activator and urokinase type plasminogen activator (u-PA), catalyze the activation of plasminogen by cleaving the $\mathrm{Arg}^{561}$ $\mathrm{Val}^{562}$ bond to yield active serine protease plasmin, which consists of two polypeptide chains that are held together

K. Hasumi (Corresponding author), K. Hasegawa, Y. Kitano: Department of Applied Biological Science, Tokyo Noko University, 3-5-8 Saiwaicho, Fuchu-shi, Tokyo 183-8509, Japan, E-mail: hasumi@cc.tuat.ac.jp by disulfide bridges [2]. Circulating plasminogen resists its activation, since it adopts tight, spiral conformation due to intramolecular binding of $\mathrm{Lys}^{50}$ and/or Lys ${ }^{62}$ to lysine binding site (aminohexyl site) in the fifth kringle domain [4 6]. Fibrin and cellular receptors bind plasminogen and relax plasminogen conformation to be highly activatable, promoting efficient localized proteolysis $[7,8]$. Similarly, lysine analogs bind to plasminogen kringles, resulting in conformational relaxation and enhancement of activation to plasmin [4]. Thus, conformational modulation of plasminogen is important for its activation.

We discovered a variety of novel nonlysine-analog modulators of plasminogen activation. These include a family of microbial triprenyl phenol metabolites designated SMTPs, which enhance both plasminogen activation and plasminogen-fibrin binding, resulting in augmentation of fibrinolysis [9 16]. These effects are strikingly different from the action of lysine analog modulators, which enhance plasminogen activation but inhibit plasminogen-fibrin binding and fibrinolysis. Therefore, SMTPs could be candidates for development of fibrinolysis-promoting agents. In the present paper, we describe the isolation of a new congener, SMTP-0 (Fig. 1), which lacks the side chain of SMTPs and permits determination of its absolute configuration by the modified Mosher method.

Previously, we found that the production of SMTPs by Stachybotrys microspora could be selectively enhanced by supplementing the culture medium with an appropriate amine, of which nitrogen atom is to be incorporated as the lactam nitrogen atom in the SMTP molecule [14]. We have improved the method by investigating the composition of production medium and the timing of amine feeding. The essential modification was to restrict the amount of amines in the production medium in the early phase of culture so as to permit cell growth but suppress the 

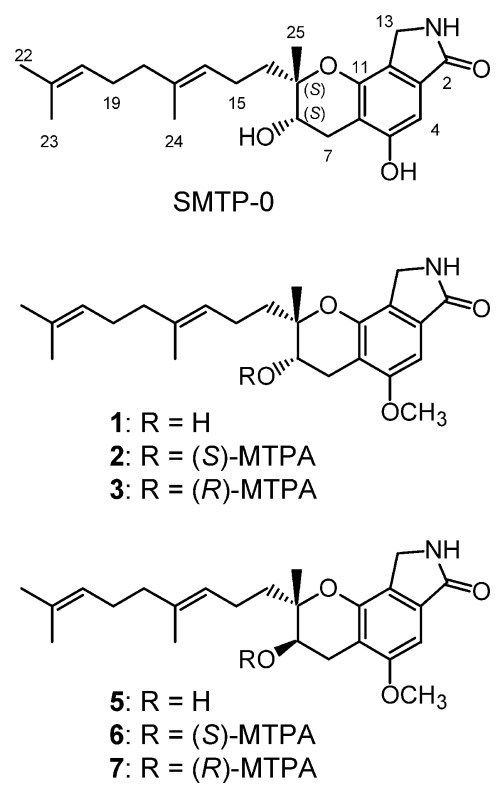

Fig. 1 Absolute structure of $\mathbf{1}, \mathbf{2}, \mathbf{3}, \mathbf{5}, \mathbf{6}$ and $\mathbf{7}$. SMTP-0, 5$O$-Me-SMTP-0 (1), 8-[S- $\alpha$-methoxy- $\alpha$-(trifluoromethyl)phenylacetoxy]-5-O-Me-SMTP-0 (2), 8-[R- $\alpha$-methoxy- $\alpha$-(trifluoromethyl)phenylacetoxy]-5-O-Me-SMTP-0 (3), 8-epi-5-O-MeSMTP-0 (5), 8-epi-8-[S- $\alpha$-methoxy- $\alpha$-(trifluoromethyl)phenyacetoxy]-5-O-Me-SMTP-0 (6), and 8-epi-8-[R- $\alpha$-methoxy$\alpha$-(trifluoromethyl)phenylacetoxy]-5-O-Me-SMTP-0 (7) are shown.

Table 1 Physico-chemical properties of SMTP-0

\begin{tabular}{ll}
\hline Appearance & White amorphous solid \\
Molecular formula & $\mathrm{C}_{23} \mathrm{H}_{31} \mathrm{NO}_{4}$ \\
MALDI-TOF-MS $(\mathrm{m} / \mathrm{z})$ & \\
$\quad$ Found $(\mathrm{M}+\mathrm{H})^{+}:$ & 386.2599 \\
$\quad$ Calcd: & 386.2331 for $\mathrm{C}_{23} \mathrm{H}_{32} \mathrm{NO}_{4}$ \\
UV $\lambda_{\max }^{\mathrm{MeOH}} \mathrm{nm}(\varepsilon)$ & $215(55,770), 251(10,620), 300(4,000)$ \\
& \\
IR $v_{\max }$ (neat) $\mathrm{cm}^{-1}$ & $3259,2918,1666,1612,1470,1360$ \\
& 1167,1082 \\
{$[\alpha]_{D}^{25}$} & $-7.4^{\circ}\left(c 0.5, \mathrm{CHCl}_{3}\right)$ \\
\hline
\end{tabular}

formation of byproducts. SMTP congeners of interest can be produced selectively by adding appropriate amines after accumulation of a triprenyl phenol precursor. SMTP-0 was produced using the improved method as described in the experimental section. From $100 \mathrm{ml}$ of the $S$. microspora IFO 30018 culture, $33.2 \mathrm{mg}$ of purified SMTP-0 was isolated.

The physico-chemical properties of SMTP-0 are shown in Table 1. The NMR and MS results (see Table 2 for NMR data) suggested that the compound retained the backbone structure common to the previously isolated SMTPs but that it lacked the $N$-linked side chain (Fig. 1). The structural formula of SMTP-0 was identical to that of stachybotrin $\mathrm{B}$, isolated by $\mathrm{Xu}$ et al. as an antimicrobial agent. However, the optical rotation of SMTP-0 was $-7.4^{\circ}$, but that of stachybotrin B was $+39.1^{\circ}$. The NMR data of stachybotrin B were quite similar to those of SMTP-0 (Table 2). These observations suggest that SMTP-0 is an enantiomer of stachybotrin B, while its absolute configuration has been unknown. So, we tried to determine the absolute configuration of SMTP-0. In the previous studies, we determined the relative stereochemistry of the dihydropyran moiety of SMTP [15]. There remained two possible combinations of absolute configuration at $\mathrm{C} 8$ and C9, i.e. $8 R, 9 R$ and $8 S, 9 S$. The modified Mosher method is known to give an absolute configuration for a chiral carbon atom with a secondary hydroxyl group $[18,19]$. Because SMTP-0 has two hydroxyl groups (one attached to the chiral carbon atom at $\mathrm{C} 8$ and the other to the aromatic carbon atom at $\mathrm{C} 5$ ), we first converted SMTP-0 to its 5-O-Me derivative (1) using trimethylsilyldiazomethane [20] (Fig. 1). Next, compound 1 was esterified using $R-(-)$ - and $S$ - $(+)-\alpha$-methoxy- $\alpha$ (trifluoromethyl)phenylacetylchloride to afford $8-[S-\alpha-$ methoxy- $\alpha$-(trifluoromethyl)phenylacetoxy]-5-O-MeSMTP-0 (2) and 8-[R- $\alpha$-methoxy- $\alpha$-(trifluoromethyl)phenylacetoxy]-5-O-Me-SMTP-0 (3), respectively (Fig. 1). The chemical shift values of ${ }^{1} \mathrm{H}-\mathrm{NMR}$ spectra for compounds $\mathbf{2}$ and $\mathbf{3}$ were compared each other (Table 3 ). Characteristic differences in the chemical shift value $\left(\Delta \delta=\delta_{S}-\delta_{R}\right)$ were observed at the axial proton at $\mathrm{C} 7$ $(\Delta \delta=-0.12)$, the aromatic proton at $\mathrm{C} 4(\Delta \delta=-0.04)$, the olefinic proton at $\mathrm{C} 16(\Delta \delta=+0.04)$ and the methyl protons at $\mathrm{C} 25(\Delta \delta=+0.05)$, thus indicating an $S$ configuration at C8. To exclude ambiguity, we further tried to synthesize Mosher esters using an epimer of SMTP-0. First, alcohol 1 was oxidized with Dess-Martin periodinane [21], affording ketone 4 . Then, ketone 4 was reduced with $\mathrm{NaBH}_{4}$ to yield racemic alcohol, from which 8-epi-5-O-Me-SMTP-0 (5) was isolated by reverse phase HPLC. Both $S$ and $R$ Mosher esters (6 and 7, respectively) were prepared from $\mathbf{5}$. As shown in Table 3, plus and minus signs of the characteristic differences in the chemical shift values between $\mathbf{6}$ and 7 were opposite as compared with the differences between 2 and 3. These results support the conclusion that configuration at C8 is $S$. Therefore, the configurations at C8 and C9 were assigned as $S$ and $S$ (Fig. 1). Because all the SMTP congeners are produced by the same strain of $S$. microspora IFO 30018 and the optical rotation of the congeners with relatively simple $N$-linked side chain, staplabin $\left[-\left(\mathrm{CH}_{2}\right)_{4} \mathrm{COOH}\right]$ and SMTP-1 $\left[-\left(\mathrm{CH}_{2}\right)_{2} \mathrm{OH}\right]$, are in the minus values $\left(-11.0^{\circ}\right.$ and $-7.1^{\circ}$, respectively) $[9$, 
Table 2 NMR spectral data for SMTP-0 and stachybotrin B

\begin{tabular}{|c|c|c|c|c|}
\hline \multirow{2}{*}{ No. } & \multicolumn{2}{|r|}{ SMTP-0 } & \multicolumn{2}{|c|}{ Stachybotrin B } \\
\hline & $\delta_{\mathrm{C}}$ & $\delta_{\mathrm{H}}$ & $\delta_{\mathrm{C}}$ & $\delta_{\mathrm{H}}$ \\
\hline 2 & 170.24 & & 174.2 & \\
\hline 3 & 131.78 & & 132.5 & \\
\hline 4 & 99.44 & $6.62(1 \mathrm{H}, \mathrm{s})$ & 100.8 & $6.74(1 \mathrm{H}, \mathrm{s})$ \\
\hline 5 & 155.93 & & 158.0 & \\
\hline 6 & 111.29 & & 113.5 & \\
\hline \multirow[t]{2}{*}{7} & 26.59 & $2.82(1 \mathrm{H}, \mathrm{dd}, J=5.5,17.4)$ & 27.8 & $2.98(1 \mathrm{H}, \mathrm{dd}, J=6,18)$ \\
\hline & & $2.45(1 \mathrm{H}, \mathrm{dd}, J=7.7,17.6)$ & & $2.64(1 \mathrm{H}, \mathrm{dd}, J=6,18)$ \\
\hline 8 & 66.01 & $3.73(1 \mathrm{H}, \mathrm{t}, J=6.4)$ & 68.4 & $3.87(1 \mathrm{H}, \mathrm{dd}, J=6)$ \\
\hline 9 & 78.52 & & 80.2 & \\
\hline 11 & 148.45 & & 150.2 & \\
\hline 12 & 121.61 & & 124.1 & \\
\hline \multirow[t]{2}{*}{13} & 41.95 & $4.09(1 \mathrm{H}, \mathrm{d}, J=16.9)$ & 44.2 & $4.20(1 \mathrm{H}, \mathrm{d}, J=18)$ \\
\hline & & $4.05(1 \mathrm{H}, \mathrm{d}, J=16.9)$ & & $4.23(1 \mathrm{H}, \mathrm{d}, J=18)$ \\
\hline 14 & 37.14 & $1.59(2 \mathrm{H}, \mathrm{m})$ & 38.5 & $1.67(2 \mathrm{H}, \mathrm{m})$ \\
\hline 15 & 20.93 & $2.10(2 \mathrm{H}, \mathrm{m})$ & 22.6 & $2.18(2 \mathrm{H}, \mathrm{m})$ \\
\hline 16 & 124.16 & $5.11(1 \mathrm{H}, \mathrm{t}, J=6.6)$ & 125.5 & $5.14(1 \mathrm{H}, \mathrm{m})$ \\
\hline 17 & 134.18 & & 136.2 & \\
\hline 18 & 39.04 & $1.91(2 \mathrm{H}, \mathrm{m})$ & 40.8 & $1.95(2 \mathrm{H}, \mathrm{m})$ \\
\hline 19 & 26.06 & $1.99(2 \mathrm{H}, \mathrm{m})$ & 27.8 & $2.02(2 \mathrm{H}, \mathrm{m})$ \\
\hline 20 & 123.98 & $5.04(1 \mathrm{H}, \mathrm{t}, J=7.1)$ & 125.3 & $5.05(1 \mathrm{H}, \mathrm{m})$ \\
\hline 21 & 130.52 & & 132.2 & \\
\hline 22 & 25.27 & $1.61(3 \mathrm{H}, \mathrm{s})$ & 25.8 & $1.63(3 \mathrm{H}, \mathrm{s})$ \\
\hline 23 & 17.36 & $1.53(3 \mathrm{H}, \mathrm{s})$ & 17.7 & $1.55(3 \mathrm{H}, \mathrm{s})$ \\
\hline 24 & 15.48 & $1.54(3 \mathrm{H}, \mathrm{s})$ & 15.7 & $1.57(3 \mathrm{H}, \mathrm{s})$ \\
\hline 25 & 17.95 & $1.15(3 \mathrm{H}, \mathrm{s})$ & 18.2 & $1.27(3 \mathrm{H}, \mathrm{s})$ \\
\hline
\end{tabular}

The chemical shift for SMTP-0 is relative to DMSO- $d_{6}\left(\delta_{\mathrm{C}} 39.5 \mathrm{ppm} ; \delta_{\mathrm{H}} 2.49 \mathrm{ppm}\right)$ and that for stachybotryn B is from ref. 17. The coupling constant $(\mathrm{J})$ is given in $\mathrm{Hz}$.

11], we believe that the absolute configuration at the dihydropyran moiety of other SMTPs is the same as that of SMTP-0.

SMTP-0 was eventually inactive in enhancing plasminogen activation when it was assayed as described previously [15] at concentrations ranging from 50 $500 \mu \mathrm{M}$, whereas SMTP-7, one of most effective congeners, enhanced the activity 10 -fold at $65 \mu \mathrm{M}$. This result clearly shows that the $N$-linked side chain of SMTP plays a significant role in exerting plasminogen activation enhancing activity. Although SMTP-0 itself is an inactive compound, it can be a potential precursor for chemical derivatization through introducing a side chain at the lactam nitrogen atom.

SMTP-0 showed antibacterial activity in disk assay against Corynebacterium ammoniagenes (IFO 12612) at 100 and $300 \mu \mathrm{g} /$ disk $(8 \mathrm{~mm})$ with an inhibitory zone of 9 and $9.5 \mathrm{~mm}$, respectively. However, the compound did not affect the growth of Bacillus subtilis (IFO 12210) and Escherichia coli (IFO 3301) at $300 \mu \mathrm{g} /$ disk. SMTP-0 also inhibited the radial growth of the fungus Rhizomucor pusillus (IFO 4579 ) by $40 \%$ in a center-point inoculation assay at $100 \mu \mathrm{g} / \mathrm{disk}$, while the compound failed to inhibit the growth of Penicillium notatum (IAM 1026) at $1 \mathrm{mg} /$ disk.

\section{Experimental}

\section{Production and Purification of SMTP-0}

S. microspora IFO 30018 was incubated at $25^{\circ} \mathrm{C}$ for 4 days in a $500-\mathrm{ml}$ Erlenmeyer flask containing $100 \mathrm{ml}$ of a seed medium consisting of glucose $4.0 \%$, soybean meal $0.5 \%$, dry bouillon $0.3 \%$, yeast extract $0.3 \%$ and the antifoam 
Table $3 \quad{ }^{1} \mathrm{H}-\mathrm{NMR}$ data for compounds 1, 2, 3, 5, 6 and $\mathbf{7}$

\begin{tabular}{|c|c|c|c|c|c|c|c|}
\hline \multirow{2}{*}{ Position } & \multicolumn{2}{|c|}{1 (5-O-Me-SMTP-0) } & \multicolumn{2}{|c|}{$\mathbf{2}$ (S -MTPA ester of $\mathbf{1}$ ) } & \multicolumn{2}{|c|}{$\mathbf{3}$ (R-MTPA ester of $\mathbf{1})$} & \multirow{2}{*}{$\begin{array}{c}\Delta \delta \\
\left(\delta_{S}-\delta_{R}\right)\end{array}$} \\
\hline & $\delta_{\mathrm{H}}$ & & $\delta_{\mathrm{H}}$ & & $\delta_{\mathrm{H}}$ & & \\
\hline 4 & 6.89 & $(1 \mathrm{H}, \mathrm{s})$ & 6.90 & $(1 \mathrm{H}, \mathrm{s})$ & 6.94 & $(1 \mathrm{H}, \mathrm{s})$ & -0.04 \\
\hline \multirow[t]{2}{*}{7} & 2.96 & $(1 \mathrm{H}, \mathrm{dd}, J=5.5,18.0)$ & 3.16 & $(1 \mathrm{H}, \mathrm{dd}, J=5.3,18.0)$ & 3.16 & $(1 \mathrm{H}, \mathrm{dd}, J=5.1,18.0)$ & 0 \\
\hline & 2.76 & $(1 \mathrm{H}, \mathrm{dd}, J=5.5,18.0)$ & 2.76 & $(1 \mathrm{H}, \mathrm{dd}, J=6.6,18.0)$ & 2.88 & $(1 \mathrm{H}, \mathrm{dd}, J=5.9,18.0)$ & -0.12 \\
\hline 8 & 3.93 & $(1 \mathrm{H}, \mathrm{m})$ & 5.29 & $(1 \mathrm{H}, \mathrm{dd}, J=5.5,6.2)$ & 5.28 & $(1 \mathrm{H}, \mathrm{t}, J=5.5)$ & 0.01 \\
\hline \multirow[t]{2}{*}{13} & 4.3 & $(1 \mathrm{H}, \mathrm{d}, J=17.2)$ & 4.27 & $(2 \mathrm{H}, \mathrm{s})$ & 4.28 & $(2 \mathrm{H}, \mathrm{s})$ & -0.01 \\
\hline & 4.26 & $(1 \mathrm{H}, \mathrm{d}, J=17.2)$ & & & & & 0 \\
\hline 14 & $\sim 1.63$ & $(2 \mathrm{H}, \mathrm{m})$ & $\sim 1.64$ & $(2 \mathrm{H}, \mathrm{m})$ & $\sim 1.63$ & $(2 \mathrm{H}, \mathrm{m})$ & 0.01 \\
\hline 15 & 2.13 & $(2 \mathrm{H}, \mathrm{m})$ & 2.12 & $(2 \mathrm{H}, \mathrm{m})$ & 2.11 & $(2 \mathrm{H}, \mathrm{m})$ & 0.01 \\
\hline 16 & 5.08 & $(1 \mathrm{H}, \mathrm{m})$ & 5.06 & $(1 \mathrm{H}, \mathrm{m})$ & 5.02 & $(1 \mathrm{H}, \mathrm{m})$ & 0.04 \\
\hline 18 & 1.94 & $(2 \mathrm{H}, \mathrm{m})$ & 1.96 & $(2 \mathrm{H}, \mathrm{t}, J=7.7)$ & 1.96 & $(2 \mathrm{H}, \mathrm{t}, J=7.7)$ & 0 \\
\hline 19 & 2.02 & $(2 \mathrm{H}, \mathrm{m})$ & 2.05 & $(2 \mathrm{H}, \mathrm{m})$ & 2.04 & $(2 \mathrm{H}, \mathrm{m})$ & 0.01 \\
\hline 20 & 5.05 & $(1 \mathrm{H}, \mathrm{m})$ & 5.07 & $(1 \mathrm{H}, \mathrm{m})$ & 5.07 & $(1 \mathrm{H}, \mathrm{m})$ & 0 \\
\hline 22 & 1.65 & $(3 \mathrm{H}, \mathrm{s})$ & 1.67 & $(3 \mathrm{H}, \mathrm{s})$ & 1.67 & $(3 \mathrm{H}, \mathrm{s})$ & 0 \\
\hline 23 & 1.55 & $(3 \mathrm{H}, \mathrm{s})$ & 1.57 & $(3 \mathrm{H}, \mathrm{s})$ & 1.56 & $(3 \mathrm{H}, \mathrm{s})$ & 0.01 \\
\hline 24 & 1.56 & $(3 \mathrm{H}, \mathrm{s})$ & 1.59 & $(3 \mathrm{H}, \mathrm{s})$ & 1.58 & $(3 \mathrm{H}, \mathrm{s})$ & 0.01 \\
\hline 25 & 1.35 & $(3 \mathrm{H}, \mathrm{s})$ & 1.28 & $(3 \mathrm{H}, \mathrm{s})$ & 1.23 & $(3 \mathrm{H}, \mathrm{s})$ & 0.05 \\
\hline 5-O-Me & 3.85 & $(3 \mathrm{H}, \mathrm{s})$ & 3.86 & $(3 \mathrm{H}, \mathrm{s})$ & 3.87 & $(3 \mathrm{H}, \mathrm{s})$ & -0.01 \\
\hline & & & $\cdots$ & $\cdots$ & $\cdots$ & $\cdots$ & . \\
\hline MTPA & & & 3.45 & $(3 \mathrm{H}, \mathrm{s})$ & 3.49 & $(3 \mathrm{H}, \mathrm{s})$ & \\
\hline \multirow[t]{3}{*}{ moiety } & & & 7.36 & $(3 \mathrm{H}, \mathrm{m})$ & 7.30 & $(2 \mathrm{H}, \mathrm{t}, J=7.7)$ & \\
\hline & & & 7.48 & $(2 \mathrm{H}, \mathrm{d}, J=7.7)$ & 7.36 & $(1 \mathrm{H}, \mathrm{t}, J=7.3)$ & \\
\hline & & & & & 7.43 & $(2 \mathrm{H}, \mathrm{d}, J=7.7)$ & \\
\hline \multirow{2}{*}{ Position } & \multicolumn{2}{|c|}{5 (8-epi-5-O-Me-SMTP-0) } & \multicolumn{2}{|c|}{6 (S -MTPA ester of $\mathbf{5}$ ) } & \multicolumn{2}{|c|}{$\mathbf{7}$ (R-MTPA ester of $\mathbf{5}$ ) } & $\Delta \delta$ \\
\hline & $\delta_{\mathrm{H}}$ & & $\delta_{\mathrm{H}}$ & & $\delta_{\mathrm{H}}$ & & $\left(\delta_{S}-\delta_{R}\right)$ \\
\hline 4 & 6.93 & $(1 \mathrm{H}, \mathrm{s})$ & 6.95 & $(1 \mathrm{H}, \mathrm{s})$ & 6.9 & $(1 \mathrm{H}, \mathrm{s})$ & 0.05 \\
\hline \multirow[t]{2}{*}{7} & 2.93 & $(1 \mathrm{H}, \mathrm{dd}, J=5.1,18.0)$ & 3.06 & $(1 \mathrm{H}, \mathrm{dd}, J=5.1,18.0)$ & 3.06 & $(1 \mathrm{H}, \mathrm{dd}, J=5.1,18.0)$ & 0 \\
\hline & 2.82 & $(1 \mathrm{H}, \mathrm{dd}, J=4.8,18.0)$ & 2.97 & $(1 \mathrm{H}, \mathrm{dd}, J=5.1,18.0)$ & 2.85 & $(1 \mathrm{H}, \mathrm{dd}, J=5.1,18.0)$ & 0.12 \\
\hline 8 & 3.94 & $(1 \mathrm{H}, \mathrm{m})$ & 5.27 & $(1 \mathrm{H}, \mathrm{t}, J=5.1)$ & 5.27 & $(1 \mathrm{H}, \mathrm{t}, J=5.5)$ & 0 \\
\hline \multirow[t]{2}{*}{13} & 4.31 & $(2 \mathrm{H}, \mathrm{s})$ & 4.28 & $(1 \mathrm{H}, \mathrm{d}, J=17.0)$ & 4.27 & $(1 \mathrm{H}, \mathrm{d}, J=17.2)$ & 0.01 \\
\hline & & & 4.26 & $(1 \mathrm{H}, \mathrm{d}, J=17.0)$ & 4.23 & $(1 \mathrm{H}, \mathrm{d}, J=17.2)$ & 0.03 \\
\hline 14 & 1.78 & $(2 \mathrm{H}, \mathrm{m})$ & 1.58 & $(2 \mathrm{H}, \mathrm{m})$ & 1.65 & $(2 \mathrm{H}, \mathrm{m})$ & -0.07 \\
\hline 15 & 2.14 & $(2 \mathrm{H}, \mathrm{m})$ & 2.03 & $(2 \mathrm{H}, \mathrm{m})$ & 2.05 & $(2 \mathrm{H}, \mathrm{m})$ & -0.02 \\
\hline 16 & 5.16 & $(1 \mathrm{H}, \mathrm{m})$ & 4.97 & $(1 \mathrm{H}, \mathrm{m})$ & 5.03 & $(1 \mathrm{H}, \mathrm{m})$ & -0.06 \\
\hline 18 & 1.98 & $(2 \mathrm{H}, \mathrm{m})$ & 1.95 & $(2 \mathrm{H}, \mathrm{m})$ & 1.95 & $(2 \mathrm{H}, \mathrm{t}, J=7.7)$ & 0 \\
\hline 19 & 2.06 & $(2 \mathrm{H}, \mathrm{m})$ & 2.00 & $(2 \mathrm{H}, \mathrm{m})$ & 2.03 & $(2 \mathrm{H}, \mathrm{m})$ & -0.03 \\
\hline 20 & 5.08 & $(1 \mathrm{H}, \mathrm{m})$ & 5.07 & $(1 \mathrm{H}, \mathrm{m})$ & 5.06 & $(1 \mathrm{H}, \mathrm{m})$ & 0.01 \\
\hline 22 & 1.67 & $(3 \mathrm{H}, \mathrm{s})$ & 1.67 & $(3 \mathrm{H}, \mathrm{s})$ & 1.66 & $(3 \mathrm{H}, \mathrm{s})$ & 0.01 \\
\hline 23 & 1.56 & $(3 \mathrm{H}, \mathrm{s})$ & 1.53 & $(3 \mathrm{H}, \mathrm{s})$ & 1.53 & $(3 \mathrm{H}, \mathrm{s})$ & 0 \\
\hline 24 & 1.60 & $(3 \mathrm{H}, \mathrm{s})$ & 1.56 & $(3 \mathrm{H}, \mathrm{s})$ & 1.56 & $(3 \mathrm{H}, \mathrm{s})$ & 0 \\
\hline 25 & 1.29 & $(3 \mathrm{H}, \mathrm{s})$ & 1.28 & $(3 \mathrm{H}, \mathrm{s})$ & 1.33 & $(3 \mathrm{H}, \mathrm{s})$ & -0.05 \\
\hline 5-O-Me & 3.87 & $(3 \mathrm{H}, \mathrm{s})$ & 3.87 & $(3 \mathrm{H}, \mathrm{s})$ & 3.85 & $(3 \mathrm{H}, \mathrm{s})$ & 0.02 \\
\hline MTPA & & & 3.44 & $(3 \mathrm{H}, \mathrm{s})$ & 3.43 & $(3 \mathrm{H}, \mathrm{s})$ & \\
\hline \multirow[t]{3}{*}{ moiety } & & & 7.26 & $(2 \mathrm{H}, \mathrm{t}, J=7.7)$ & 7.34 & $(3 \mathrm{H}, \mathrm{m})$ & \\
\hline & & & 7.34 & $(1 \mathrm{H}, \mathrm{m})$ & 7.45 & $(2 \mathrm{H}, \mathrm{d}, J=7.7)$ & \\
\hline & & & 7.39 & $(2 \mathrm{H}, \mathrm{d}, J=7.7)$ & & & \\
\hline
\end{tabular}


CB442 (Nippon Oil \& Fat Co.) 0.01\%, pH 5.8. The seed culture $(5.0 \mathrm{ml})$ was transferred to a $500-\mathrm{ml}$ Erlenmeyer flask containing $100 \mathrm{ml}$ of medium consisting of sucrose $5.0 \%$, yeast extract $0.1 \%, \mathrm{NaNO}_{3} 0.3 \%, \mathrm{~K}_{2} \mathrm{HPO}_{4} 0.1 \%$, $\mathrm{MgSO}_{4} \cdot 7 \mathrm{H}_{2} \mathrm{O} 0.05 \%$, $\mathrm{KCl} 0.05 \%, \mathrm{CoCl}_{2} \cdot 6 \mathrm{H}_{2} \mathrm{O} 0.00025 \%$, $\mathrm{FeSO}_{4} \cdot 7 \mathrm{H}_{2} \mathrm{O} 0.0015 \%, \mathrm{CaCl}_{2} \cdot 2 \mathrm{H}_{2} \mathrm{O} 0.00065 \%$ and $\mathrm{CB} 442$ $0.01 \%, \mathrm{pH} 5.8$. The flask was incubated at $25^{\circ} \mathrm{C}$ on a rotary shaker at $180 \mathrm{rpm}$. After 96 hours, $100 \mathrm{mg}$ of ammonium chloride was added, and the flask was incubated further for 24 hours. The culture was stopped by adding $200 \mathrm{ml}$ of $\mathrm{MeOH}$. The $\mathrm{MeOH}$ extracts were filtered and concentrated to remove $\mathrm{MeOH}$. The residue was extracted thrice with an equal volume of ethyl acetate. The ethyl acetate extracts were combined and concentrated to dryness, dissolved in $3.0 \mathrm{ml}$ of $\mathrm{MeOH}$, treated with Lichrolut ${ }^{\circledR} \mathrm{RP}-18$ (Merck), and subjected to purification by preparative HPLC using an Inertsil PREP-ODS $(30 \times 250 \mathrm{~mm}$; GL Science, Tokyo, Japan). The column was developed at a rate of $25 \mathrm{ml} /$ minute at $40^{\circ} \mathrm{C}$ with $50 \mathrm{mM}$ ammonium acetate in $75 \%$ aqueous $\mathrm{MeOH}$. SMTP-0 was eluted at a retention time of 11.5 minutes. Fractions containing SMTP-0 were evaporated to remove $\mathrm{MeOH}$ and extracted with ethyl acetate, giving $33.2 \mathrm{mg}$ of purified material.

\section{5-O-Me-SMTP-0 (1)}

SMTP-0 (34 mg) in $2.0 \mathrm{ml}$ of methanol - acetonitrile $(1: 9$, $\mathrm{v} / \mathrm{v})$ was mixed with $200 \mu \mathrm{l}$ of trimethylsilyldiazomethane ( $2 \mathrm{M}$ in $n$-hexane) and $40 \mu \mathrm{l}$ of $N, N$-diisopropylethylamine at room temperature for 24 hours. The mixture was concentrated and passed through a short silica gel column developed with ethyl acetate, affording $27 \mathrm{mg}$ of $\mathbf{1}$. MALDI-TOF-MS, $m / z 399.2410(\mathrm{M}+\mathrm{H})^{+}$; optical rotation, $[\alpha]_{\mathrm{D}}^{25}-5.9^{\circ}\left(c 1.0, \mathrm{CHCl}_{3}\right) ;{ }^{1} \mathrm{H}-\mathrm{NMR}$, see Table 3.

\section{8-[S- $\alpha$-Methoxy- $\alpha$-(trifluoromethyl)phenylacetoxy $]-5-O$ - Me-SMTP-0 (2)}

Compound $1(12 \mathrm{mg}, 30 \mu \mathrm{mol})$ in $1.0 \mathrm{ml}$ of dichloromethane was mixed with $10 \mu \mathrm{l}$ of triethylamine, $2.0 \mathrm{mg}$ of $4-$ dimethylaminopyridine and $15 \mathrm{mg}(60 \mu \mathrm{mol})$ of $R-(-)-\alpha-$ methoxy- $\alpha$-(trifluoromethyl)phenylacetylchloride for 24 hours at room temperature. After the mixture was concentrated, the residue was subjected to preparative silica gel TLC (ethyl acetate) to isolate $15 \mathrm{mg}$ of $\mathbf{2}$. MALDITOF-MS, $m / z 616.2976(\mathrm{M}+\mathrm{H})^{+}$; optical rotation, $[\alpha]_{\mathrm{D}}^{25}$ $+18.8^{\circ}\left(c 0.24, \mathrm{CHCl}_{3}\right) ;{ }^{1} \mathrm{H}-\mathrm{NMR}$, see Table 3.

\section{8-[R- $\alpha$-Methoxy- $\alpha$-(trifluoromethyl)phenylacetoxy]-5- $O$-Me-SMTP-0 (3)}

Starting from $12 \mathrm{mg}$ ( $30 \mu \mathrm{mol})$ of $\mathbf{1}, 15 \mathrm{mg}$ of compound $\mathbf{3}$ was prepared as described above using $S$-( -$)$ - $\alpha$-methoxy$\alpha$-(trifluoromethyl)phenylacetylchloride $(15 \mathrm{mg}, 60 \mu \mathrm{mol})$ instead of the $R$ enantiomer. MALDI-TOF-MS, $\mathrm{m} / \mathrm{z}$ $616.2943(\mathrm{M}+\mathrm{H})^{+}$; optical rotation, $[\alpha]_{\mathrm{D}}^{25}+41.9^{\circ}($ c 0.35 , $\left.\mathrm{CHCl}_{3}\right) ;{ }^{1} \mathrm{H}-\mathrm{NMR}$, see Table 3.

\section{8-epi-5-O-Me-SMTP-0 (5)}

To a solution of alcohol $1(60 \mathrm{mg}, 150 \mu \mathrm{mol})$ in $5.0 \mathrm{ml}$ of $\mathrm{CH}_{2} \mathrm{Cl}_{2}, 96 \mathrm{mg}(226 \mu \mathrm{mol})$ Dess-Martin periodinane was added. The reaction mixture was stirred at ambient temperature for 2 hours, and the reaction was quenched with a $1: 1$ solution of saturated aq $\mathrm{Na}_{2} \mathrm{~S}_{2} \mathrm{O}_{3}$ and saturated aq $\mathrm{NaHCO}_{3}(40 \mathrm{ml})$. The mixture was extracted with $\mathrm{CH}_{2} \mathrm{Cl}_{2}$ (30 ml, thrice). The combined organic extracts were washed with water, dried over $\mathrm{MgSO}_{4}$, filtered, and concentrated to yield ketone 4 that was used immediately in the next step. $\mathrm{NaBH}_{4}(15 \mathrm{mg}, 400 \mu \mathrm{mol})$ was added to a solution of ketone $\mathbf{4}$ in $\mathrm{MeOH}(5.0 \mathrm{ml})$. The reaction mixture was stirred at ambient temperature for 1 hour and was then added brine $(50 \mathrm{ml})$. The mixture was extracted with EtOAc ( $30 \mathrm{ml}$, thrice). The combined organic extracts were washed with water, dried over $\mathrm{MgSO}_{4}$, filtered, and concentrated to yield a $1: 3$ mixture of $\mathbf{1}$ and $\mathbf{5}$. The mixture was subjected to purification by reversed-phase HPLC [Inertsil PREP-ODS, $30 \times 250 \mathrm{~mm}$, GL Science, developed at $12.5 \mathrm{ml} /$ minute with a linear gradient of aq $\mathrm{MeOH}$ ( $80 \sim 90 \%$ for 40 minutes) containing $0.1 \%$ formic acid] to give pure 5 (4.8 mg). MALDI-TOF-MS, $\mathrm{m} / \mathrm{z}$ $400.1589(\mathrm{M}+\mathrm{H})^{+}$; optical rotation, $[\alpha]_{\mathrm{D}}^{25}-29.5^{\circ}($ c 0.39 , $\left.\mathrm{CHCl}_{3}\right) ;{ }^{1} \mathrm{H}-\mathrm{NMR}$, see Table 3.

\section{8-epi-8-[S- $\alpha$-Methoxy- $\alpha$-(trifluoromethyl)phenylacetoxy]- 5-O-Me-SMTP-0 (6)}

A solution of alcohol $5(2.3 \mathrm{mg}, 5.8 \mu \mathrm{mol})$ in $\mathrm{CH}_{2} \mathrm{Cl}_{2}$ $(1.0 \mathrm{ml})$ was mixed with triethylamine $(10 \mu \mathrm{l})$, 4dimethylaminopyridine $(1.0 \mathrm{mg})$, and $(R)-\alpha$-methoxy- $\alpha$ (trifluoromethyl)phenylacetyl chloride $(5.0 \mathrm{mg}, 20 \mu \mathrm{mol})$. The reaction mixture was stirred at ambient temperature for 18 hours and was then concentrated. The mixture was subjected to preparative TLC (100\% ethyl acetate) to afford purified 6 (3.2 mg). MALDI-TOF-MS, $m / z 616.2969$ $(\mathrm{M}+\mathrm{H})^{+}$; optical rotation, $[\alpha]_{\mathrm{D}}^{25}-47.8^{\circ}\left(c 0.25, \mathrm{CHCl}_{3}\right)$; ${ }^{1} \mathrm{H}-\mathrm{NMR}$, see Table 3.

\section{8-epi-8-[R- $\alpha$-Methoxy- $\alpha$-(trifluoromethyl)phenylacetoxy]- 5-O-Me-SMTP-0 (7)}

Starting from $2.3 \mathrm{mg}(5.8 \mu \mathrm{mol})$ of alcohol $5,3.0 \mathrm{mg}$ of compound 7 was prepared as described above using $S$-(-)$\alpha$-methoxy- $\alpha$-(trifluoromethyl)phenylacetylchloride $(5.0 \mathrm{mg}, 20 \mu \mathrm{mol})$ instead of the $R$ enantiomer. MALDITOF-MS, $m / z 616.2972(\mathrm{M}+\mathrm{H})^{+}$; optical rotation, $[\alpha]_{\mathrm{D}}^{25}$ $-38.4^{\circ}$ ( $\left.0.25, \mathrm{CHCl}_{3}\right)$; ${ }^{1} \mathrm{H}-\mathrm{NMR}$, see Table 3. 


\section{General}

UV spectrum was measured in $\mathrm{MeOH}$ on a model 320 spectrometer (Hitachi, Tokyo, Japan) and IR spectrum on a JIR-WINSPEC spectrometer (JEOL, Tokyo, Japan) with $\mathrm{NaCl}$. MALDI-TOF-MS spectrum was taken on a Voyager DE STR spectrometer (Applied Biosystem, CA, USA) using $\alpha$-cyano-4-hydroxycinnamic acid as a matrix. NMR spectra were measured in DMSO- $d_{6}$ or $\mathrm{CDCl}_{3}$ on an EX270 or an Alpha-600 spectrometer (JEOL). Optical rotation was measured on a model DIP-360 (JASCO, Tokyo Japan) in $\mathrm{CHCl}_{3}$.

Acknowledgments The authors thank Teppei Miura for chemical synthesis of the SMTP-0 derivatives.

\section{References}

1. Carmeliet P, Collen D. Molecular genetics of the fibrinolytic and coagulation systems in haemostasis, thrombogenesis, restenosis and atherosclerosis. Curr Opin Lipidol 8: 118-125 (1997)

2. Kolev K, Machovich R. Molecular and cellular modulation of fibrinolysis. Thromb Haemost 89: 610-621 (2003)

3. Forsgren M, Raden B, Israelsson M, Larsson K, Heden LO. Molecular cloning and characterization of a full-length cDNA clone for human plasminogen. FEBS Lett 213: 254-260 (1987)

4. Marshall JM, Brown AJ, Ponting CP. Conformational studies of human plasminogen and plasminogen fragments: evidence for a novel third conformation of plasminogen. Biochemistry 33: 3599-3606 (1994)

5. An SS, Carreno C, Marti DN, Schaller J, Alberico F, Llinas M. Lysine-50 is a likely site for anchoring the plasminogen $N$-terminal peptide to lysine-binding kringles. Protein Sci 7: 1960-1969 (1998)

6. Cockell CS, Marshall JM, Dawson KM, CederholmWilliams SA, Ponting CP. Evidence that the conformation of unliganded human plasminogen is maintained via an intramolecular interaction between the lysine-binding site of kringle 5 and the $N$-terminal peptide. Biochem J 333: 99-105 (1998)

7. Nesheim, ME, Fredenburgh JC, Larsen GR. The dissociation constants and stoichiometries of the interactions of Lys-plasminogen and chloromethyl ketone derivatives of tissue plasminogen activator and the variant $\triangle F E I X$ with intact fibrin. J Biol Chem 265: 21541-21548 (1990)

8. Hajjar KA, Nacman RL. Endothelial cell-mediated conversion of Glu-plasminogen to Lys-plasminogen. Further evidence for assembly of the fibrinolytic system on the endothelial cell surface. J Clin Invest 82: 1769-1778 (1988)

9. Shinohara C, Hasumi K, Hatsumi W, Endo A. Staplabin, a novel fungal triprenyl phenol which stimulates the binding of plasminogen to fibrin and U937 cells. J Antibiot 49: 961-966 (1996)

10. Takayasu R, Hasumi K, Shinohara C, Endo A. Enhancement of fibrin binding and activation of plasminogen by staplabin through induction of a conformational change in plasminogen. FEBS Lett 418: 58-62 (1997)

11. Kohyama T, Hasumi K, Hamanaka A, Endo A. SMTP-1 and -2, novel analogs of staplabin produced by Stachybotrys microspora IFO30018. J Antibiot 50: 172-174 (1997)

12. Hasumi K, Ohyama S, Kohyama T, Ohsaki Y, Takayasu R, Endo A. Isolation of SMTP-3, -4, -5 and -6, novel analogs of staplabin, and their effects on plasminogen activation and fibrinolysis. J Antibiot 51: 1059-1068 (1998)

13. $\mathrm{Hu} \mathrm{W}$, Ohyama S, Hasumi K. Activation of fibrinolysis by SMTP-7 and -8, novel staplabin analogs with a pseudosymmetric structure. J Antibiot 53: 241-247 (2000)

14. $\mathrm{Hu} \mathrm{W}$, Ohyama S, Narasaki R, Hasumi K. Selective production of staplabin and SMTPs in cultures of Stachybotrys microspora fed with precursor amines. J Antibiot 54: 962-966 (2001)

15. Hu W, Kitano Y, Hasumi K. SMTP-4D, -5D, -6D, -7D and $-8 \mathrm{D}$, a new series of the non-lysine-analog plasminogen modulators with a D-amino acid moiety. J Antibiot 56: 832-837 (2003)

16. Ohyama S, Harada T, Chikanishi T, Miura Y, Hasumi K. Nonlysine-analog plasminogen modulators promote autocatalytic generation of plasmin(ogen) fragments with angiostatin-like activity. Eur J Biochem 271: 809-820 (2004)

17. $\mathrm{Xu}$ X, De Guzman FS, Gloer JB, Shearer CA. Stachybotrins $\mathrm{A}$ and B: novel bioactive metabolites from a brackish water isolate of the fungus Stachybotrys sp. J Org Chem 57: 6700-6703 (1992)

18. Ohtani I, Kusumi T, Kashman Y, Kakisawa H. High-field FT NMR application of Mosher's method. The absolute configurations of marine terpenoids. J Am Chem Soc 113: 4092-4096 (1991)

19. Dale JA, Dull DL, Mosher HS. $\alpha$-Methoxy- $\alpha$-trifluoromethylphenylacetic acid, a versatile reagent for the determination of enantiomeric composition of alcohols and amines. J Org Chem 34: 2543-2549 (1969)

20. Aoyama T, Terasawa S, Sudo K, Shioiri T. New methods and reagents in organic synthesis. 46. Trimethylsilyldiazomethane: a convenient reagent for the $O$-methylation of phenols and enols. Chem Pharm Bull 32: 3759-3760 (1984)

21. Dess DB, Martin JC. A useful 12-I-5 triacetoxyperiodinane (the Dess-Martin periodinane) for the selective oxidation of primary or secondary alcohols and a variety of related 12-I-5 species. J Am Chem Soc 113: 7277-7287 (1991) 